NOTICE WARNING CONCERNING COPYRIGHT RESTRICTIONS:

The copyright law of the United States (title 17, U.S. Code) governs the making of photocopies or other reproductions of copyrighted material. Any copying of this document without permission of its author may be prohibited by law. 
AN INTEGRAL - INTERPOLATORY ITERATIVE METHOD

FOR THE SOLUTION OF NON-LINEAR SCALAR EQUATIONS

Boles law Kacewicz

Department of Computer Science

Carnegie-Mellon University

Pittsburgh, PA. 15213

(Visiting from the University of Warsaw)

January 1975 
This paper deals with the iterative solution of non-linear equations $f(x)=0$. We consider integral information on $f$ which is given by $f\left(x_{0}\right), f^{\prime}\left(x_{0}\right), \ldots, f(s)\left(x_{0}\right)$ and $\int_{0}^{x} f(t) d t$. We define an interpolatory-integral method which uses integral information and which has maximal order of convergence equal to $s+3$. Since the maximal order of iterations which use $f\left(x_{0}\right), \ldots, f(s)\left(x_{0}\right)$ is equal to $s+1$, the additional information given by the integral $\int_{0}^{x_{0}} f(t) d t$ increases the order by two.

1. INTRODUCTION

We consider the solution of the nonlinear scalar equation

(1.1) $f(x)=0$,

where $f$ is a complex function of complex variable.

In most papers which deal with stationary iterative methods for (1.1) it is assumed we know the standard information for $f$ (Wozniakowski [74])

$$
\Re_{s}=\left\{f\left(x_{0}\right), \ldots, f^{(s)}\left(x_{0}\right)\right\}
$$

where $s \geq 1$ and $x_{0}$ is an approximation to the solution $\alpha$. The maximal order of convergence of such methods is equal to $s+7$ (Traub [64], Wozniakowski [73]). We raise the question how other types of information can be used in iterative processes and what is the maximal order formation. This paper deals with integral information which consists of the standard information $\mathfrak{R}_{s}$ and addi-
tionally the value of an integral. Thus

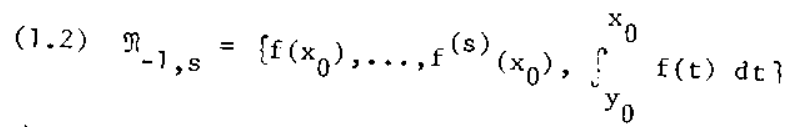

where $\mathrm{y}_{0}$ is a complex number defined in Section 3.

In Section 2 we define an interpolatory - integral method $I_{-1, s}$ which uses integral information $\Re_{-1, s}$ to estimate $\alpha$ and in Section 7 we prove $i t s$ order for $s \geq 1$ is maximal. Sections 4,5 and 6 conWozniakowski [74] defined for the general,

it is equal to the maximal order of convergence. chosen $y_{0}, p\left(\Re_{-1, s}\right)=s+3$. Since $p\left(\Re_{s}\right)=s+1$, the additional information for $s \geq 1$ and for suitable es the order of information by two. and will be reported to a future paper. 
2. INTERPOLATORY - INTEGRAL ITERATIVE METHOD I $-1, s$

Let us consider the solution of the nonlinear equation,

(2.1) $f(x)=0$,

where $f: D \rightarrow \mathbb{C}, D$ is an open subset of $\mathbb{C}, \mathbb{C}$ denotes the set of complex numbers. Let $\alpha \in D$ be $a$ simple zero of $\mathrm{f}, \mathrm{f}(\alpha)=0 \neq \mathrm{f}^{\prime}(\alpha)$. An interpolatory - integral method $\mathrm{I}_{-1, \mathrm{~s}}$ is defined as follows. Let $x_{i}$ be an approximation of $\alpha$. We assume that the information on $f$ is given by

(2.2) $\mathfrak{\eta}_{-1, s}=\mathfrak{N}_{-1, s}\left(\mathrm{x}_{i} ; f\right)=\left\{f\left(\mathrm{x}_{i}\right), \ldots, f^{(s)}\left(\mathrm{x}_{i}\right), \int_{y_{i}}^{x_{i}} f(t) d t\right\}$, where $y_{i}$ depends on $x_{i}, f^{(k)}\left(x_{i}\right), k=0,1, \ldots, s, y_{i} \neq x_{i}$ and is defined in Section 3 . If $s=0$ then $y_{i}$ can depend on $x_{i}, f\left(x_{i}\right), x_{i-1}, f\left(x_{i-1}\right)$. The value of $y_{i}$ will be chosen to maximize the order of $i t e r a-$ tion.

The information consists of the standard information given by $f\left(x_{i}\right), \ldots, f^{(s)}\left(x_{i}\right)$ and additionally the value of the integral. Next, let $w_{i}$ be an interpolatory polynomial of degree at most $s+1$ such that:

$(2.3) w_{i}^{(k)}\left(x_{i}\right)=f^{(k)}\left(x_{i}\right) \quad k=0,1, \ldots, s$,

(2.4) $\int_{y_{i}}^{x_{i}} w_{i}(t) d t=\int_{y_{i}}^{x_{i}} f(t) d t$

If $w_{i}$ exists then the next approximation $x_{i+1}$ in $I_{-1, s}$ method is defined as a zero of polynomial $w_{i}$,

$$
(2.5) \quad w_{i}\left(x_{i+1}\right)=0 \text {, }
$$

with a criterion to make $x_{i+1}$ unique. We shall now prove that $w_{i}$ exists and is unique. Let

(2.6) $F(x)=\int_{y_{i}}^{x} f(t) d t$,

and let $g_{i}$ be a polynomial of degree $\leq s+2$ such that

(2.7) $g_{i}\left(y_{i}\right)=F\left(y_{i}\right)=0$,

(2.8) $g_{i}^{(k)}\left(x_{i}\right)=F^{(k)}\left(x_{i}\right), \quad k=0,1, \ldots, s+1$.

Thus, $g_{i}$ is a Hermite interpolatory polynomial for $F$. The assumption $y_{i} \neq x_{i}$ implies the existence and the uniqueness of $\mathrm{g}$. Set

(2.9) $w_{i}(x)=g_{i}^{\prime}(x)$. 

Then (2.3) and (2.4) are satisfied which completes the proof. Moreover, from (2.8) and (2.9) follows an
error formula,

$(2.10) \quad F(x)-g_{i}(x)=\left(x-y_{i}\right)\left(x-x_{i}\right)^{s+2} G_{i}(x)$

where

(2.11) $G_{i}(x)=G_{i}(x, f)=\int_{0}^{1} \int_{0}^{t} \cdots \int_{0}^{t} s+2 f(s+2)\left(y_{i}+t_{j}\left(x_{i}-y_{i}\right)+t_{s+3}\right.$.

$\left.\left(x-x_{i}\right)\right) d t_{1} \ldots d t_{s+3}$.

Differentiating $(2.10)$ we get from $(2.11)$,

(2.12) $f(x)-w_{i}(x)=R(x)$

where

$R(x)=R(x, f)=\left(x-x_{i}\right)^{s+1}\left\{\left[(s+2)\left(x-y_{i}\right)+x-x_{i}\right] G_{i}(x)+G_{i}^{\prime}(x)\left(x-x_{i}\right)\left(x-y_{i}\right)\right\}$

3. DEFINITION OF A LOWER LIMIT OF THE INTEGRAL

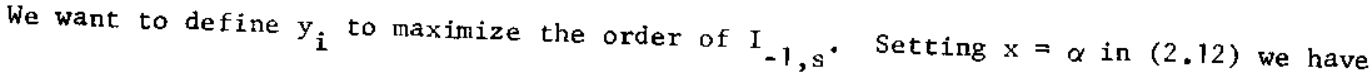
(3.1) $-w_{i}(\alpha)=R(\alpha)$

Let us assume for a moment that $w_{i}$ has a zero $x_{i+1}$ sufficiently close to a simple zero $\alpha$.

$$
\mathrm{x}_{i+1}-\alpha=-\frac{w_{i}(\alpha)}{w_{i}^{i}(\alpha)}+o\left(\left(x_{i+1}-\alpha\right)^{2}\right)=O(R(\alpha))
$$

We see that the order of iteration depends mainly on $R(\alpha)$. Therefore we shall choose $y_{i}$ to minimize
$R(\alpha)$ in a certain sense. From (2.12)

(3.2) $R(\alpha)=\left(\alpha-x_{i}\right)^{s+1}\left[\left[(s+2)\left(\alpha-y_{i}\right)+\alpha-x_{i}\right] G_{i}(\alpha)+G_{i}^{\prime}(\alpha)\left(\alpha-x_{i}\right)\left(\alpha-y_{i}\right)\right.$.

As $G_{i}(\alpha)$ and $G_{i}^{\prime}(\alpha)$ are in general unknown we want to minimize

(3.3) $\max \left(\left|(s+2)\left(\alpha-y_{i}\right)+\alpha-x_{i}\right|,\left|\left(\alpha-x_{i}\right)\left(\alpha-y_{i}\right)\right|\right)$.

One can verify that the minimal value of (3.3) is for $y_{i}$ equal to $y$

(3.4) $y=\alpha+\frac{\because-x_{i}}{s+2+\mid \alpha-x_{i} T}$ 
As we do not know $\alpha$ we have to replace it by an approximation to $\alpha$, $z$ which depends only on the standard information, $z_{i}=z_{i}\left(x_{i}, f\left(x_{i}\right), \ldots, f(s)\left(x_{i}\right)\right)$ and $z_{i} \neq x_{i}$. If $s=0$ then $z_{i}=z_{i}\left(x_{i-1}, f\left(x_{i-1}\right), x_{i}, f\left(x_{i}\right)\right)$. We define $y_{i}$ as

(3.5) $y_{i}=z_{i}+\frac{z_{i}-x_{i}}{s+2+\left|z_{i}-x_{i}\right|}$.

It can be proved that one can drop $\left|z_{i}-x_{i}\right|$ in the denominator without the change of the order. Finally, $y_{i}$ is defined by

(3.6) $y_{i}=z_{i}+\frac{z_{i}-x_{i}}{s+2}$

Hence, from $(3.6)$ and $(2.12)$ we get

(3.7) $f(x)-w_{1}(x)=R(x)$

for

$$
R(x)=R(x, f)=\left(x-x_{i}\right)^{s+1}\left\{(s+3)\left(x-z_{i}\right) G_{i}(x)+G_{i}^{+}(x)\left(x-x_{i}\right) \frac{(s+3)\left(x-z_{i}\right)+x_{i}-x}{s+2}\right\}
$$

where $w_{i}$ is the interpolatory polynomial defined in section 2 .

4. THE CONVERGENCE OF THE ITERATIVE METHOD $I_{-1, s}$ FOR $s \geq 1$

previous section we have seen that the order of iteration mainly depends on $R(\alpha)$. From

$$
R(\alpha)=\left(\alpha-x_{i}\right)^{s+1}\left\{(s+3)\left(\alpha-z_{i}\right) G_{i}(\alpha)+G_{i}^{\prime}(\alpha)\left(\alpha-x_{i}\right) \frac{(s+3)\left(\alpha-z_{i}\right)+x_{i}-\alpha}{s+2}\right.
$$

Hence, to assure the maximal order of $I_{-1, s}$ for $s \geq 1$ it suffices to define approximation $z_{i}$ using Newton method

(4.1) $z_{i}-z_{i}\left(x_{i}, f\left(x_{i}\right), \ldots, f(s)\left(x_{i}\right)\right)=x_{i}-\frac{f\left(x_{i}\right)}{f^{\prime}\left(x_{i}\right)} \quad i=0,1, \ldots$

\section{Theorem I}

If $s \geq 1$ and

1. $\mathrm{f}^{(\mathrm{s}+3)}$ is a continuous function on $\mathrm{K}(\alpha, \mathrm{R})$ where

$$
\begin{aligned}
& \mathrm{f}(\alpha)=0 \neq \mathrm{f}^{\prime}(\alpha) \\
& \mathrm{K}(\alpha, \mathrm{R})=\{\mathrm{x}:|\mathrm{x}-\alpha| \leq \mathrm{R}\}, \mathrm{R}=\mathrm{R}(\Gamma)=\max \left(\frac{(\mathrm{s}+3) \mathrm{C} \Gamma^{2}+\Gamma, \Gamma}{\mathrm{s}+2}\right)
\end{aligned}
$$

where the constant $C$ is defined below, 
2. a real number $\Gamma 0$ is such that

for

$$
\begin{gathered}
\Gamma h(\Gamma)<1 \text { and } \frac{2 M_{2}}{v_{1}} \Gamma<1 \text { where } \\
h(\Gamma)=\frac{2}{v_{1}}(2 \Gamma)^{s}\left\{(1+c \Gamma) \frac{M_{s+2}}{(s+2) !}+\frac{2 M_{s+3}}{(s+4) !} \frac{(s+3)\left(\Gamma+c \Gamma^{2}\right)+2 \Gamma}{s+2}\right\}
\end{gathered}
$$

$$
\begin{gathered}
v_{1}=\inf _{x \in J}\left|\frac{f(x)}{x-\alpha}\right| ; M_{i}=\sup _{x \in K(\alpha, R)}\left|f^{(i)}(x)\right| \\
M_{2}=\sup _{x \in J}\left|f^{\prime \prime}(x)\right| ; C=C(\Gamma)=\frac{M_{2}}{2 v_{1}} \frac{1}{\left(1-\frac{M_{2}}{v_{1}} \Gamma\right)^{2}} ;
\end{gathered}
$$$$
i=s+2, s+3
$$

3. $x_{0} \in J$ where $J=\{x:|x-\alpha| \leq \Gamma\}$,

then the sequence $\left\{x_{i}\right\}$ generated in $I_{-1, s}$ has the following properties:

(i) $x_{i} \in J, \forall_{i}$,

(ii) $x_{i+1}-\alpha=A_{i}\left(x_{i}-\alpha\right)^{s+3}, \forall i$,

where

$\left|A_{i}\right| \leq A \quad \forall i$, and

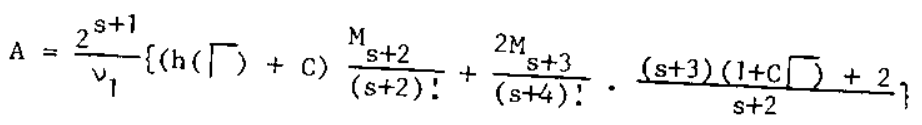

(iii) $\lim _{i \rightarrow \infty} x_{i}=\alpha \quad$ and

$\lim _{i \rightarrow \infty} \frac{x_{i+1}-\alpha}{\left(x_{i}-\alpha\right)^{s+3}}=B \quad$ where

$B=(-1)^{s+2}\left\{\frac{f^{\prime \prime}(\alpha) \cdot f^{(s+2)}(\alpha)}{2\left(f^{\prime}(\alpha)\right)^{2}(s+2) !}+\frac{f^{(s+3)}(\alpha)}{(s+4) !} \frac{1}{f^{\prime}(\alpha)(s+2)}\right\}$

Proof of (i) (By induction).

Let us assume that $x_{i} \in J$. From $(2.12) w_{i}(x)=0$ iff $x=H(x)$ where

(4.2) $H(x)= \begin{cases}\alpha+\frac{1}{\frac{f(x)}{x-\alpha}} R(x) & \text { if } x \neq \alpha \\ \alpha+\frac{1}{f^{\prime}(\alpha)} R(\alpha) & \text { if } x=\alpha\end{cases}$ 
Now, using $(4.2),(3.7),(2.11)$ and condition 2 one can verify that the assumptions of the Brouwer fixpoint theorem hold for $H$ in the set $J$. Hence, there exists $x \in J$, such that $w_{i}(x)=0$. So $x_{i+1} \in J$ and, by induction, $x_{i} \in J i=0,1, \ldots$.

Proof of (ii)

From $(4.2)$ and $(3.7)$ we get

$$
x_{i+1}-\alpha=\frac{1}{\frac{f\left(x_{i+1}\right)}{x_{i+1}-\alpha}} R\left(x_{i+1}\right) \quad i=0,1, \ldots
$$

Therefore

$$
\begin{aligned}
& \text { (4.3) } x_{i+1}-\alpha=A_{i}\left(x_{i}-\alpha\right)^{s+3} \quad \text { where } \\
& A_{i}=\frac{1}{\frac{f\left(x_{i+1}\right)}{e_{i+1}}}\left(\frac{e_{i+1}}{e_{i}}-1\right)^{s+1}\left\{(s+3)\left(\frac{e_{i+1}}{e_{i}}-c_{i}\right) G\left(x_{i+1}\right)+\right. \\
& \left.+G^{\prime}\left(x_{i+1}\right)\left(\frac{e_{i+1}}{e_{i}}-1\right)-\frac{(s+3)\left(\frac{e_{i+1}}{e_{i}}-c_{i} e_{i}\right)+1-\frac{e_{i+1}}{e_{i}}}{s+2}\right) \\
& z_{i}-\alpha=C_{i}\left(x_{i}-\alpha\right)^{2}
\end{aligned}
$$

An upper bound on $A_{i}$ one can find using an assumption 2 and (2.11).

\section{Proof of (iii)}

From (4.3) and (i),

$$
\left|x_{i+1}-\alpha\right| \leq A\left|x_{i}-\alpha\right|^{s+3}
$$

and thus

$$
\left|x_{i+1}-\alpha\right| \leq\left(A \Gamma^{s+2}\right)^{i+1}\left|x_{0}-\alpha\right| \quad \forall i
$$

From assumption 2 follows that $A \Gamma^{s+2}<1$, and hence,

$$
\lim _{i \rightarrow \infty} x_{i}=\alpha
$$

Finally from $(4.3)$ and $(4.4)$ it can be shown that

$$
\lim _{i \rightarrow \infty} \frac{x_{i+1}-\alpha}{\left(x_{i}-\alpha\right)^{s+3}}=\lim _{i \rightarrow \infty} A_{i}=B
$$

which completes the proof of Theorem 1. 
In general, B is not equal zero (see point (iii) which means that $s+3$ is the order of the interpolatory - integral method $I_{-1, s}$ for $s \geq 1$, (Traub [64], Wozniakowski [74]). Note that iterative methods which use only the standard information $f\left(x_{i}\right), \ldots, f^{(s)}\left(x_{i}\right)$ have orders at most s+l. Additional information given by $\int_{y}^{x_{i}} f(t) d t$ increases the order of $I_{-1, s}$ by two. The usage of the $I_{-1, s}$ method in practice is profitable if the evaluation cost of the value of integral is approximately equal to the evaluation cost of function or its derivatives.

5. THE CONVERGENCE OF THE I $-1,0$ ME THOD

Now, we assume $s=0$, which means that the information is of the form:

$$
\mathfrak{N}_{-1,0}=\left\{f\left(x_{i}\right), \int_{y_{i}}^{x} f(t) d t\right\} \text { where } y_{i}=z_{i}+\frac{z_{i}-x_{i}}{2} .
$$

Note that we cannot now define $z_{i}$ by the Newton method as we do not know the value of the first derivative. Let $z_{i}$ be given now by the secant method,

(5.1) $z_{i}=x_{i}-\frac{x_{i}-x_{i-1}}{f\left(x_{i}\right)-f\left(x_{i-1}\right)} f\left(x_{i}\right) \quad \forall_{i}$

In this case, the interpolatory - integral method $I_{-1,0}$ is a one-point method with memory (Traub [64]).

\section{Theorem 2}

If

1. $\mathrm{f}^{(3)}$ is a continuous function on $\mathrm{K}(\alpha, \mathrm{R})=\{\mathrm{x}:|\mathrm{x}-\alpha| \leq \mathrm{R}\}$, where $f(\alpha)=0 \neq \mathrm{f}^{\prime}(\alpha)$

$$
R=R(\Gamma)=\max \left(\frac{3 C^{-2}+\Gamma}{2}, \Gamma\right)
$$

where the constant $C$ is defined below,

2. a real number $\Gamma \cdot 0$ is such that

where

$$
\Gamma \cdot h(\Gamma)<1 \text { and } \frac{2 M_{2}}{\nu_{1}} \Gamma<1,
$$

for

$$
h(\Gamma)=\frac{2}{v_{1}}\left\{(1+c \Gamma) \cdot \frac{M_{2}}{2}+\frac{M_{3}}{24}\left[3\left(\Gamma+c \Gamma^{2}\right)+2 \Gamma\right]\right\}
$$

$$
\begin{aligned}
& \nu_{1}=\inf _{x \in J}\left|\frac{f(x)}{x-\alpha}\right|, M_{i}=\sup _{x \in K(\alpha, R)}\left|f^{(i)}(x)\right| \quad i=2,3 ; \\
& M_{2}^{\prime}=\sup _{x \in J}\left|f^{\prime \prime}(x)\right|, C=C(\Gamma)=\frac{M_{2}^{\prime}}{2 v_{1}} \cdot \frac{1}{\left(1-\frac{M_{2}^{\prime}}{v_{1}} \Gamma\right)^{2}}
\end{aligned}
$$


3. $x_{0}, x_{1} \in J$ where $J=\left\{x:|x-\alpha| \leq\lceil\}\right.$, then the sequence $\left\{x_{i}\right\}$, generated in $I_{-1,0}$ has the following properties:

$$
\begin{aligned}
& \text { (i) } x_{i} \in J \quad i=0,1, \ldots \\
& \text { (ii) } x_{i+1}-\alpha=A_{i}\left(x_{i}-\alpha\right)^{2}\left(x_{i-1}-\alpha\right) \\
& \text { where }\left|A_{i}\right| \leq A \quad i=0,1, \ldots, \\
& A=\frac{2}{\nu_{1}}\left\{(h(\Gamma)+C) \frac{M_{2}}{2}+\frac{M_{3}}{24}[3(1+c \mid)+2]\right\}, \\
& \text { (iii) } \lim _{i \rightarrow \infty} x_{i}=\alpha \quad \text { and moreover } \\
& \lim _{i \rightarrow \infty} \frac{x_{i+1}-\alpha}{\left(x_{i}-\alpha\right)^{2}\left(x_{i-1}-\alpha\right)}=B \quad \text { where B }=\frac{1}{4}\left(\frac{f^{\prime \prime}(\alpha)}{f^{\prime}(\alpha)}\right)^{2}, \\
& \text { (iv) } \lim _{i \rightarrow \infty} \frac{x_{i+1}-\alpha}{x_{i}-\left.\alpha\right|^{P}}=B^{p^{+1}} \text { where } p=1+\sqrt{2} .
\end{aligned}
$$

The proof of this theorem is onitted since it is similar to the proof of Theorem 1 . From (iv) follows that $1+\sqrt{2}$ is the order of the interpolatory - integral method $I_{-1,0^{\circ}}$

6. THE CONVERGENCE OF THE $I_{-1, s}$ METHOD FOR MULTIPLE ZEROS

Let us now assume that $s \geq 1$ and $\alpha$ is an $m$ fold zero, i.e.,

$$
f(\alpha)=f^{\prime}(\alpha)=\ldots \ldots=f^{(m-1)}(\alpha)=0 \neq f^{(m)}(\alpha)
$$

where $m \leq s$.

The information is given by

$$
\begin{gathered}
\mathfrak{x}_{-1, s}=\left\{f\left(x_{i}\right), f^{\prime}\left(x_{i}\right), \ldots, f^{(s)}\left(x_{i}\right), \int_{y_{i}}^{x_{i}} f(t) d t\right\} \text { where } \\
y_{i}=z_{i}+\frac{z_{i}-x_{i}}{s+2} \text { for } z_{i}=I_{0, s}\left(x_{i} ; f\right)
\end{gathered}
$$

The notation $I_{0, s}\left(x_{i} ; f\right)$ is used by Traub [64] and Wozniakowski [73]. The character of convergence of the $I_{-1, s}$ method in this case is given by Theorem 3 .

Theorem 3

$$
\text { If } s \geq 1 \text { and }
$$

1. $f^{(s+3)}$ is a continuous function on $K(\alpha, R)=\{x:|x-\alpha| \leq R\}$ where 
where the constant $D$ is defined below,

$$
R=R(\Gamma)=\max \left(\frac{(s+3) D \Gamma^{\frac{s+m}{m}}+\Gamma}{s+2}, \Gamma\right)
$$

2. a real number $\Gamma>0$ is such that

$$
\begin{aligned}
& \text { (i) } \Gamma^{\frac{\mathrm{s}+\mathrm{I}}{\mathrm{m}}-1} \cdot \mathrm{h}(\Gamma)<1, \\
& \text { where }
\end{aligned}
$$

$h(\Gamma)=\frac{m}{v_{m}} 2^{\frac{s+1}{m}}\left\{\left(\Gamma+D \Gamma^{\frac{s+1}{m}}\right) \frac{M_{s+2}}{(s+2) !}+\frac{M_{s+3}}{(s+4) !} \cdot \frac{2 \Gamma}{s+2}\left[(s+3)\left(\Gamma+D \Gamma^{\frac{s+1}{m}}\right)+2 \Gamma\right]\right\}^{\frac{1}{m}}$,

$$
\nu_{m}=\inf _{x \in J}\left|\frac{f(x)}{(x-\alpha)^{m}}\right|, M_{s+1}=\sup _{x \in J}\left|f^{(s+1)}(x)\right|
$$

$$
M_{i}=\sup _{x \in K(\alpha, R)}\left|f^{(i)}(x)\right| \quad i=s+2, s+3,
$$

and

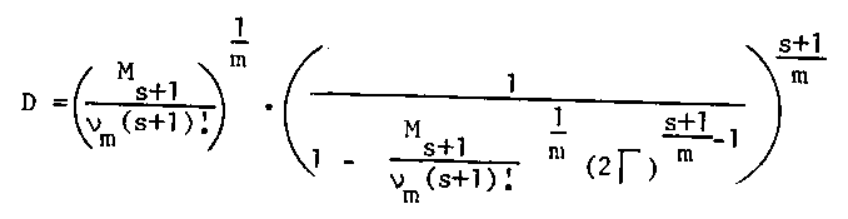

3. $x_{0} \in J, J=\{x:|x-\alpha| \leq \Gamma\}$

then

$$
\begin{aligned}
& \text { (i) } x_{i} \in J, i=0,1, \ldots, \lim _{i \rightarrow \infty} x_{i}=\alpha \\
& \text { (ii) }\left|x_{i+1}-\alpha\right| \leq A_{i}\left|x_{i}-\alpha\right|^{\frac{s+1+p}{m}} \text { where } p=\min \left(\frac{s+1}{m}, 2\right) . \\
& \text { Moreover }
\end{aligned}
$$

$$
\begin{aligned}
& A_{i} \leq A \\
& A=\sqrt[m]{\frac{1}{v_{m}}} 2^{\frac{s+1}{m}}\left\{\Gamma^{\frac{s+1}{m}-p}(h(\Gamma)+D) \frac{M_{s+2}}{(s+2) !}+\Gamma^{-2-p} \frac{M s+3}{(s+4) !} \cdot \frac{2(s+3)\left(1+D \Gamma^{\frac{s+1}{m}-1}\right)+4}{s+2}\right\}^{\frac{1}{m}},
\end{aligned}
$$


(iii) $\lim _{i \rightarrow \infty} \frac{\left|x_{i+1}-\alpha\right|}{\left|x_{i}-\alpha\right|^{\frac{s+1+p}{m}}} \leq B$ where

$$
\begin{aligned}
& B=\sqrt[m]{\frac{m !}{\left|f^{(m)}(\alpha)\right|}}\left\{\zeta_{\frac{s+1}{m}-p, 0}\left(\frac{\left|f^{(s+1)}(\alpha)\right| \cdot m !}{\left|f^{(m)}(\alpha)\right| \cdot(s+1) !}\right)^{\frac{1}{m}} \frac{\left.\mid f^{(s+2)}(\alpha)\right\rfloor}{(s+2) !}+\zeta_{2-p, 0} \frac{\left.\mid f^{(s+3)}(\alpha)\right\rfloor}{(s+4) !} \cdot \frac{1}{s+2}\right\}^{\frac{1}{m}}, \\
& s_{i, 0}=\left\{\begin{array}{lll}
0 & \text { if } & \text { i } \neq 0 \\
1 & \text { if } & i=0
\end{array}\right.
\end{aligned}
$$

(iv) $p(m)=(s+1+p) / m$ is the order of convergence of $I_{-1, s}$ method for $m$ multiple zeros.

The proof of this theorem is omitted since it is similar to the proof of Theorem 1.

7. MAXIMALITY OF I $-1, \mathrm{~s}$

Let $\Psi_{-1, \text { s }}$ be a class of stationary iterative methods $\varphi_{-1, s}$ which use information $\Re_{-1, s}$ and which have well defined order $p\left(\varphi_{-1, s}\right)$ (Woznlakowski [74]). From Theorem 1 it follows that the interpolatoryintegral method $I_{-1, s}$ belongs to $\Psi_{-1, s}, s \geq 1$.

Now we shall prove that $I_{-1, s}$ has maximal order in the class $\Psi_{-1, s}$, i.e.,

$$
P\left(I_{-1, s}\right)=\varphi_{-1, s} \sup _{-1, s} P\left(\varphi_{-1, s}\right)
$$

Wozniakowski [74] defined (Definition 7) the order of information $p(\mathfrak{N})$ and proved it is equal to the maximal order. Thus, it suffices to show that in our case

$$
\left.p\left(I_{-1, s}\right)=p^{(\Re,-1, s}\right) .
$$

Theorem 4

$$
\text { Let } n_{-1, s}=n_{-1, s}\left(x_{i} ; f\right)=\left\{f\left(x_{i}\right), \ldots, f(s)\left(x_{i}\right), \int_{y_{i}}^{x_{i}} f(t) d t\right\} \text {, for any } y_{i}=y_{i}\left(x_{i}, f\left(x_{i}\right), \ldots, f(s)\left(x_{i}\right)\right) \text {. }
$$
Then

$$
p\left(\Re_{-1, s}\right) \leq s+3 .
$$

If $s \geq 1$ and $y_{i}=z_{i}+\frac{z_{i}-x_{i}}{s+2}$ for $z_{i}=x_{i}-\frac{f\left(x_{i}\right)}{f^{\prime}\left(x_{i}\right)}$

then $P(\mathfrak{R}-1, s)=s+3$.

Proof

Let $\mathcal{F}$ be the class of complex functions of complex variable which have a simple zero and which 
are analytic in the neighborhood of $\alpha$ (see Definition 1 in Wozniakowski [74]). We recall the definition of the order of information. Let $f \in \mathcal{F}$ and $\left\{f_{i}\right\} \subset \mathcal{F}$ where

$$
f(\alpha)=0
$$

(7.1) $f_{i}\left(\alpha_{i}\right)=0, \quad i=0,1, \ldots, \lim _{i \rightarrow \infty} \alpha_{i}=\alpha$

(7.2) $\lim _{i \rightarrow \infty} f_{i}^{(k)}(\alpha)=g^{(k)}(\alpha)$, for $k=0,1, \ldots, g \in \xi, g(\alpha)=0$

Next let us assume that

(7.3) $\mathbb{R}_{-1, s}\left(\mathrm{x}_{i}, f\right)=\mathfrak{N}_{-1, s}\left(\mathrm{x}_{i}, \mathrm{f}_{i}\right) \quad \forall i$, where $\left\{x_{i}\right\}$ is an arbitrary sequence converging to $\alpha$. Let $w_{i}$ be an interpolatory polynomial of degree at
most $s+1$ defined as follows:

Thus

$$
\mathfrak{N}_{-1, s}\left(x_{i}, w_{i}\right)=\mathfrak{N}_{-1, s}\left(x_{i}, f\right), \quad \text { vi. }
$$

$$
f(x)-f_{i}(x)=f(x)-w_{i}(x)+w_{i}(x)-f_{i}(x)=R(x, f)-R\left(s, f_{i}\right) .
$$

From it and from $(2.12)$ it follows

$$
\begin{aligned}
(7.4) & \alpha-\alpha_{i}=o\left(\left(f(\alpha)-f_{i}(\alpha)\right)\right)=o\left(|R(\alpha, f)|+\left|R\left(\alpha, f_{i}\right)\right|\right)= \\
& =o\left(\left|\alpha-x_{i}\right|^{s+1} \cdot\left|(s+2)\left(\alpha-y_{i}\right)+\alpha-x_{i}\right|+\left|\alpha-x_{i}\right|^{s+2} \cdot\left|\alpha-y_{i}\right|\right)
\end{aligned}
$$

Moreover, we shall show that this bound is sharp. Let $\zeta$ be a number defined as follows:

Let

$$
\zeta= \begin{cases}1 & \text { if } \lim _{i \rightarrow \infty} \frac{\alpha-y_{i}}{\alpha-x_{i}}=-\frac{1}{s+2} \\ 0 & \text { otherwise }\end{cases}
$$

where

$$
h_{i}(x)=\left(x-x_{i}\right)^{s+1+\zeta}\left(x-b_{i}\right) \quad i=0,1, \ldots
$$

Setting

$$
b_{i}=\frac{(s+2+\zeta) y_{i}+x_{i}}{s+3+5}
$$

(7.5) $\quad f_{i}(x)=f(x)+h_{i}(x), \quad \forall i$

for any function $f \in \mathcal{F},\left(f(\alpha)=0, f_{i}\left(\alpha_{i}\right)=0\right)$ one can verify that conditions (7.1), (7.2) and (7.3) hold. Next, there exist constants $c_{i} 0$ such that $\lim _{i \rightarrow \infty} C_{i}=C \quad 0$ and 
(7.6) $\left|\alpha-\alpha_{i}\right|=c_{i}\left|f(\alpha)-f_{i}(\alpha)\right|=c_{i}\left|\alpha-x_{i}\right|^{s+1+\zeta}\left|\alpha-b_{i}\right|=\frac{c_{i}}{s+3+\zeta}\left|\alpha-x_{i}\right|^{s+1+\zeta}\left|(s+2+\zeta)\left(\alpha-y_{i}\right)+\alpha-x_{i}\right|$

which proves that $(7.4)$ is sharp.

From (7.4) and (7.6) it follows that for any $y_{i}$ the order of information $p=p\left(\Re_{-1, s}\right)$ exists. Let us assume that $p>s+3$. Let $\varepsilon>0$ be a number such that $p-\varepsilon>s+3+\varepsilon$. For $f$ and $\left\{f_{j}\right\}$ given by (7.5) we get from (7.6):

$$
+\infty>\lim _{i \rightarrow \infty} \sup \frac{\left|\alpha-\alpha_{i}\right|}{\left|x_{i}-\alpha\right|^{p-\varepsilon}}>\lim _{i \rightarrow \infty} \sup \frac{\left|\alpha-\alpha_{i}\right|}{\left|x_{i}-\alpha\right|^{s+3+\varepsilon}}=+\infty
$$

which is a contradiction. Hence $p \leq s+3$ for any $y_{i}=y_{i}\left(x_{i}, f\left(x_{i}\right) \ldots f(s)\left(x_{i}\right)\right.$. Now we sha11 show that the above estimation of $p$ is achievable for $s \geq 1$.

Indeed, setting

$$
y_{i}=z_{i}+\frac{z_{i}-x_{i}}{s+2}, z_{i}=x_{i}-\frac{f\left(x_{i}\right)}{f^{\dagger}\left(x_{i}\right)}
$$

we have

$$
\left.\lim _{i \rightarrow \infty} \frac{\alpha-y_{i}}{\alpha-x_{i}}=-\frac{1}{s+2} \quad \text { (so } \zeta=1\right) \text {. }
$$

It is easy to verify that from $(7.4)$ and $(7.6)$ it follows

$$
p\left(\mathfrak{N}_{-1, \mathrm{~s}}\right)=\mathrm{s}+3
$$

which completes the proof of Theorem 3 .

From Theorems 1 and 3 we get

\section{Corollary 1}

The interpolatory - integral method $I_{-1, s}$ is maximal, i。e.,

$$
\mathrm{p}\left(I_{-1, s}\right)=\mathrm{p}\left(\mathfrak{R}_{-1, \mathrm{~s}}\right)
$$

References

Traub [64]

Wozniakowski [73]

J. F. Traub, Iterative Methods for Solutions of Equations, Prentice-Ha11, 1964 。

H. Wozniakowski, "Maximal Stationary Iterative Methods for the Solution of Operator " STAM J Numer Anal Vol 11, No. 5, October 1974. Also available as a Carnegie-Melion Computer Science Department report, 1973.

Wozniakowski [74]

17. Wozniakowski, "Generalized Information and Maximal Order of Iteration for Operator . Wur Also available as a Carnegie-MeIlon Computer Science Department report, 1974 . 